\title{
Primary Retroperitoneal Hydatidosis with Cholelithiasis; Management Dilemma: A Case Report.
}

\author{
Rajesh Chaudhary ${ }^{1}$,Somraj Mahajan ${ }^{2}$, Ram Kishan Abrol ${ }^{3}$, Ankit Shukla ${ }^{4}$, \\ Umesh Dhiman $^{5}$, Vikrant Dharwal ${ }^{6}$ \\ ${ }^{1}$ MS , Senior Resident ,Department. Of Surgery,Dr. R.P. Govt. Medical College Kangra At Tanda HP, India. \\ ${ }^{2}$ MS, Assistant Professor, Department. Of Surgey.Dr. R.P. Govt. Medical College Kangra At Tanda HP, India \\ ${ }^{3}$ MS, Professor,Department. Of Surgey.Dr. R.P. Govt. Medical College Kangra At Tanda HP, India \\ ${ }^{4} \mathrm{MS}$, Senior Resident, Department. Of Surgey.Dr. R.P. Govt. Medical College Kangra At Tanda HP, India. \\ ${ }^{5} \mathrm{MS}$,Department. Of Surgery, Civil Hospital Dehra, HP, India. \\ ${ }^{6} \mathrm{MBBS}$, Junior Resident, Department. Of Surgery,Dr. R.P. Govt. Medical College Kangra At Tanda HP, India.
}

\begin{abstract}
Hydatidosis is an endemic disease in sheep rearing countries around the world. Mostly hydatid cysts are seen in liver and lungs. Primary retroperitoneal hydatid cyst is a very rare disease and it's occurrence with cholelithiasis poses a management dilemma. The approach in cholelithiasis is laparoscopic cholecystectomy while retroperitoneal hydatid cyst is removed laparoscopally or through open surgical approach. Both should be operated separately with emphasis on special care to prevent of implantationof hydatid cysts in the abdominal cavity. Albendazole is the drug of choice which has been used preoperatively to sterilise the cysts as well as post operatively to prevent recurrences after surgery.
\end{abstract}

Keywords: Hydatid cyst, Echinococcus granulosus, Primary retroperitoneal hydatidosis, Antihelminths

\section{Introduction}

Hydatidosis is a zoonotic disease endemic in the sheep rearing countries around the world. Echinococcus granulosus and Echinococcus multilocularis are the causative agents of this disease in human beings. Dogs ,foxes and wolves are the definitive host of this tapeworm while sheep are the intermediate hosts. Humans are accidental hosts and a dead end for the organism. ${ }^{1}$ Hydatid cysts are formed most commonly in liver and lungs but they have been reported from other parts of the body too, including retroperitoneal space. Retroperitoneal hydatid cysts are almost always secondary to rupture of hydatid cyst of liver or surgery for the liver hydatid cyst. A hydatid cyst found in the retroperitoneum without any evidence of hydatid cyst anywhere else in the body is known as primary retroperitoneal hydatid cyst. It is an extremely rare condition with reported incidence of about $0.8 \%$. These patients are usually asymptomatic and because the hydatid cysts are extremely slow growing they are detected late when they attain enormous sizes and present with the signs of local compression. ${ }^{2}$ Presence of cholelithiasis along with hydatid disease of the retroperitoneum further complicates the picture. Treatment of hydatid cyst is mainly surgical followed by antihelminthic drugs and in selected cases puncture aspiration instillation and reaspiration(PAIR) can be done.

\section{Case report}

We present the case of a 73 years old male who presented to the outpatient department with the complaints of repeated attacks of colicky pain in right hypochondrium radiating to back and shoulder for over six months. The history of last attack of acute pain was about three weeks back. It lasted for two hours and was relieved after taking painkillers at a local hospital. There was no history of jaundice or any chronic illness in the past. Blood biochemical investigations and haematological investigations were within normal limit. Ultrasonogram of the abdomen was suggestive of a stone of size 8.8 milimeter in the gall bladder neck (picture 1). But there was a well defined heterogeneous lesion superior to upper pole of kidney measuring about 10x 8 $\mathrm{x} 7$ centimeters $(\mathrm{CM})$ in retroperitonel space in right hypochondrium, with multiple internal cystic spaces and calcification (picture 2)It was separate from right kidney and suprarenal gland. Contrast enhanced CT scan of abdomen was suggestive of a primary retroperitoneal hydatid cyst displacing the right kidney inferolaterally . Patient was started on Albendazole $15 \mathrm{mg} / \mathrm{kg}$ body weight per day in two divided doses and advised to undergo laparoscopic cholecystectomy first and then cystectomy for retroperitoneal hydatid cyst. Since the patient belonged to a very far flung area she wanted both the surgeries to be done in a single go. Hence open cholcystectomy was done and simultaneously hydatid cyst was approached transperitoneally through the same right subcostal incision (picture 3) . Cyst was adherent to the vertebrae medially and was abutting the inferior vena cava hence enucleation of the cyst after instillation of ten percent betadine with partial cystectomy, was 
done. Patient made an uneventful recovery and was discharged on tenth post operative day. Patient is still in follow up after two months of surgery and is still continuing Albendazole .

\section{Discussion}

Hydatid disease is endemic in the sheep rearing areas throughout the world especially in countries around the mediterranian sea, Australia, north Africa, south America and Australia. Few cases have been reported from Canada and united states of America (USA) where the definitive host has been fox and wolves whereas caribou has been the intermediate host. This is a disease caused by the larval stage of a cestode, Echinococcus granulosus and Echinococcus multilocularis in humans. The tapeworm resides inside the dog which is the definitive host, whereas the larval stage of this worm resides inside the sheep or cattle, which is its intermediate host. The intermediate hosts are grazing herbivores which ingest the eggs with the contaminated vegetable material. Embryo liberated in the duodenum of the intermediate host then penetrates the small intestinal wall to reach the portal circulation. Liver is the first filter where most of the larvae will settle down to form the hydatid cysts. But sometimes they bypass the liver and get lodged into lungs. Canine carnivore eats the infected organs of the animal and hence ingests the larval stage. Here it matures into adult tapeworms inside the intestine of definitive host where they produce eggs and shed them to again contaminate the herbage. This completes the life cycle of tapeworm. Humans are accidental intermediate host where the parasite follows the same fate to go to liver (63\%), lungs $(25 \%)$, muscle(5\%), bone(3\%), kidney (2\%), spleen (1\%), brain $(1 \%)$. Other sites including retroperitoneum comprises less than $1 \%{ }^{3}$ The involvement of retroperitoneum is almost always secondary to hydatid cysts of liver. These may be secondry to traumatic rupture of hydatid cyst or implantation during surgery for hydatid cyst of the liver. Sometimes protoscolices can bypass the liver and lung to reach retroperitoneum. ${ }^{2}$ They may also spread through the lymphatogenous route through the gastrointestinal tract. Although first case was reported in 1958 by Lockhart and Sapinza, since then there have been a few cases of primary retroperitoneal hydatidosis reported worldwide. The case we have presented above had gallstones to further add to the confusion. This probably is the first such case in literature. They need to be differentiated from mesenteric cyst and intestinal duplication cysts. ${ }^{4}$ The growth rate of hydatid cysts is very slow, about 1-30 milimeter ( $\mathrm{mm})$ per year and the symptoms of disease may remain suppressed for very long time. ${ }^{5}$ Retroperitoneal hydatids are usually asymptomatic. Symptoms are usually because of compression or displacement of the adjacent organs. In case of liver hydatid they may rupture into the biliary tree and cause jaundice or cholangitis . Chronic weight loss, malaise , and wasting are common features. ${ }^{1}$ Our patient had presented with billiary colic but there was no history of jaundice. The ultrasound was suggestive of cholelithiasis along with retroperitoneal hyadatidosis in right hypochondrium and right lumbar area. Ultrasound is the good initial investigation which has a specificity of nearly $90 \%$. It can show the site of origin, size and number of the cysts along with the internal contents.

World Health Organisation (WHO) classification has been based on sonographic features as was the classification proposed by Gharbi and associates which also gave the morphological characteristics of the hydatid cyst. ${ }^{6}$ Computerised tomogram (CT) scan provides a better visualisation of the cysts giving useful information about the depth and extent of the cysts along with its relationship to the adjoining structures. Thus it is helpful in planning surgery. It is $90-100 \%$ accurate for diagnosing hydatid cyst. ${ }^{4}$ Serological tests are highly useful adjuncts along with radiological tests for confirming the diagnosis of hydatidosis. Commonly used complement fixation test is positive in $65 \%$ of the patients while indirect hemagglutination test and enzyme linked immunosorbent assay(ELISA) are positive in $85 \%$ patients. ${ }^{1}$ IgE and peripheral eosinophilia can also help in diagnosis along with plain abdominal radiograph and magnetic resonance imaging (MRI). Although fine needle aspiration cytology is highly specific but rarely done due to the inherent risk of severe allergic reactions and anaphylactic shock. Although it may be done in patients where the immunological tests are negative and radiological findings are doubtful. ${ }^{7}$ The treatment modalities are medical, surgical or puncture aspiration instillation and reaspiration(PAIR). Albendazole has been used as the antihelminthic agent of choice alongwith prazequintal and mebendazole. It can be given preoperatively to sterilize the hydatid cysts in about $90 \%$ cases when given for over a month's period. However it does not prevent recurrences. It is also used as adjuvant therapy following surgery and when surgery is not feasible. Surgery is the modality of choice in retroperitoneal and liver hydatid when possible. The goals of treatment are complete removal of the parasite, preventing spillage of contents, and limiting mortality and morbidity. Aspiration of the contents during laparotomy and instillation of scolicidal agents is required to prevent the recurrences. ${ }^{8}$ Although the rate of reccurence is about 2 $\%$ in patients after liver hydatid surgery it is more for retroperitoneal hydatidosis. The patients may be prescribed antihelminthics for a longer duration and may be kept under follow up. ${ }^{9}$ The management dilemma in this case was which approach to take. Laparoscopic cholecystectomy is the gold standard for cholelithiasis. ${ }^{10}$ Whether laparoscopic or open approach but retroperitoneal hydatidosis should be dealt separately. ${ }^{11}$ As we know the spillage of hydatid fluid can result in recurrence at a later stage, every attempt should be made to prevent the spillage during surgery and even after the successful surgical cure, patients should be kept in 
constant follow up. The developed world has adopted some changes in lifestyle which has resulted in significant decrease in the hydatid disease. Children in schools should be educated about personal hygiene. Washing of hands as well as vegetable matter properly before consumption should be encouraged and practised. Entrails of the slaughterd animals should not be fed to dogs. Intimacy and frequency of contact with dogs should be curtailed especially in children. ${ }^{12}$

\section{Conclusion}

Primary retroperitoneal hydatidosis is extremely rare and the presence of associated disease complicates the picture. Which surgical approach should be taken is also difficult to decide when the patients are not willing for multiple surgeries and are difficult to follow up. Absence of disease anywhere else in the body makes it possible that there may be some other route of spread other than traumatic rupture or surgical implantation of the hydatid cyst. Laparoscopic cholecystectomy is the gold standard for gall stone disease. Retroperitoneal hydatid cyst should be approached through different incision to prevent the secondary implantation of the hydatid cysts. Although incidence of primary retroperitoneal hydatidosis is very very low but every new case being reported is going to help us understand this entity in a better way.

Picture 1.

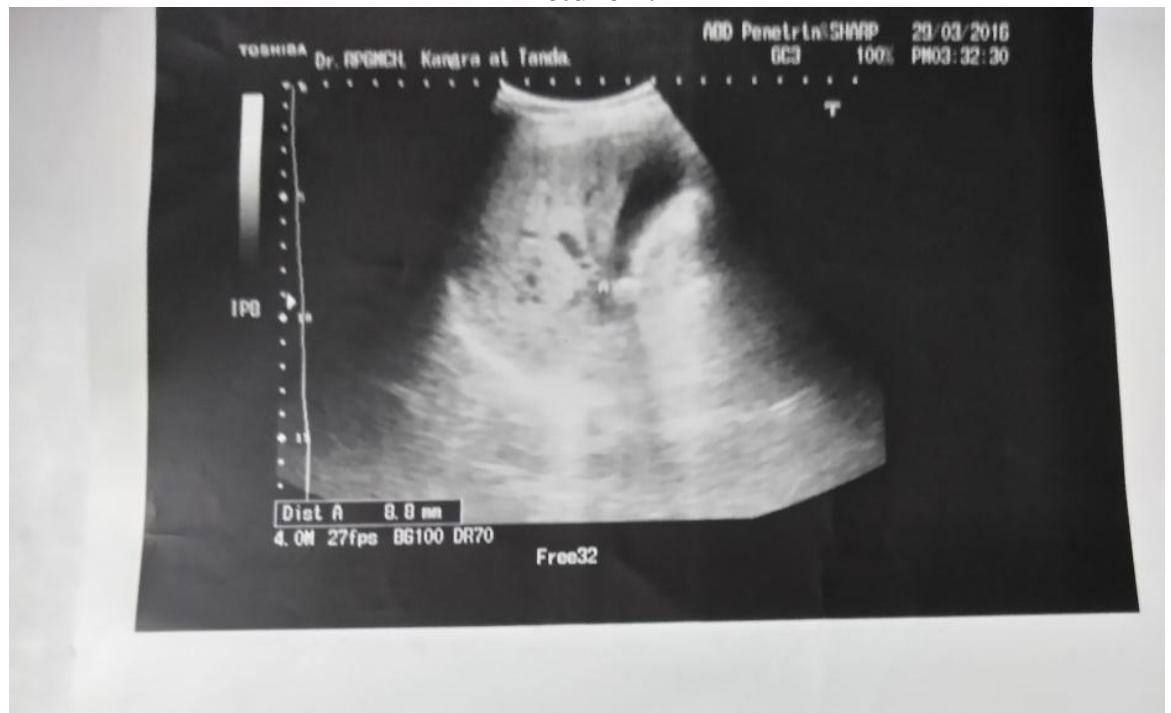

USG image showing gall bladder stone at neck.

\section{Picture 2.}

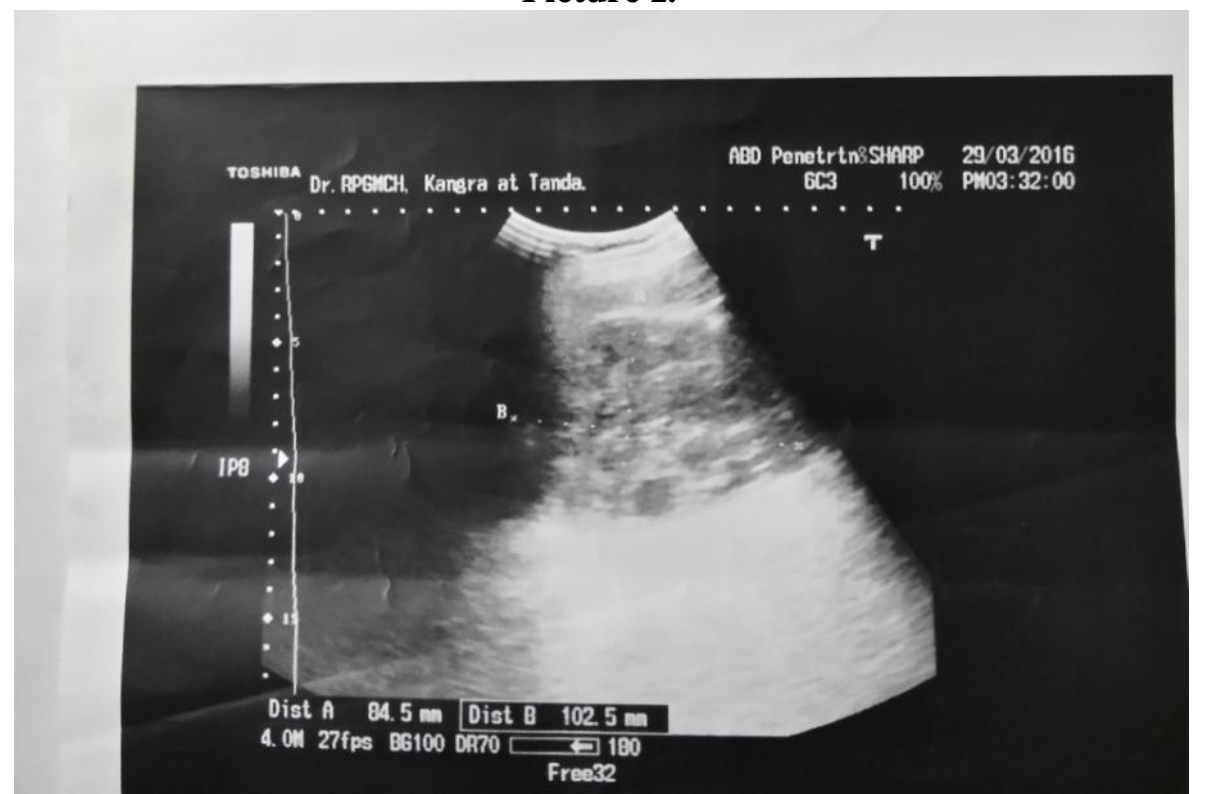

USG image showing heterogenous cystic lesion suggestive of retroperioneal hydatid cyst. 


\section{Picture 3.}

E

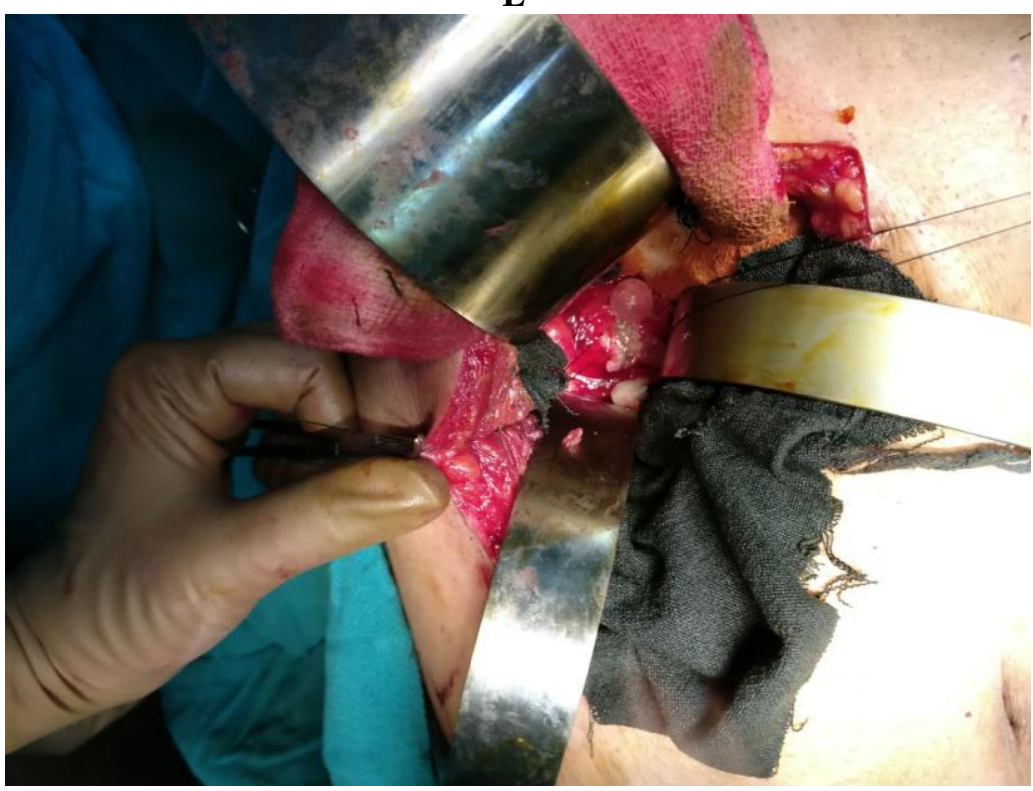

Showing daughter cysts being extracted from the retroperitoneal hydatid cyst after sterilisation of the cyst with ten percent betadine solution

\section{Conflict Of Interest}

Authors declare no conflict of interests.

\section{Consent}

Written informed consent was obtained from the patient. A copy of the written consent is available for review by the Editor-in-Chief of the journal.

\section{Author's Contributions}

Rajesh Chaudhary: Contributed substantially to the Conception, design, Acquisition of data, Analysis and interpretation of data, drafting the article, Critical revision of the article and final approval of the version to be published.

Ram Kishan Abrol: Contributed substantially to the Conception and design, Acquisition of data, Critical revision of the article and final approval of the version to be published

Ankit Shukla: Contributed substantially to the Conception and design, Acquisition of

data, Critical revision of the article and final approval of the version to be published.

Umesh Dhiman: Contributed substantially to the Conception and design, Acquisition of data, Critical revision of the article and final approval of the version to be published.

Somraj Mahajan: Contributed substantially to the Conception and design, Acquisition of data, Critical revision of the article and final approval of the version to be published.

Vikrant Dharwal: Contributed substantially to the Conception and design, Acquisition of data, Critical revision of the article and final approval of the version to be published.

Nil

\section{Acknowledgements}

\section{References}

[1]. Geller DA.(2010) Liver.In: Schwartz SI and Brunicardi CF (Eds.), Schwartz Principles of Surgery. $\left(9^{\text {th }}\right.$ edn.), McGraw-Hill Health Pub. Division, New York, USA, pp. 2083-2159.

[2]. Porwal R, Gupta HP, Singh A, Singh RK. Queer localizations of primary hydatid disease: experience from a single institute. Int Surg J 2016;3:1538-42.

[3]. McGreevy, P.B., and Nelson, G.S.: Larval cestode infections. In Strickland, G.T. (ed.): Hunter's Tropical Medicine, 6th ed. Philadelphia, W.B. Saunders, 1984, p. 771

[4]. Jain AKC, Mohan LN, Prahalad ST: Primary retroperitoneal hydatid cyst .Arch Clin Exp Surg 2016;X:X-X doi:10.5455/aces.20160114125924

[5]. WHO Informal Working Group on Echinococcosis in Humans. Bull World Health Organ 1996; 74:231-242

[6]. Gharbi HA, Hassine W, Brauner MW et al. Ultrasound examination of hydatic liver. Radiology 1981;139:459 
[7]. $\quad$ von Sinner W.N., Nyman R., Linjawi T., et al: Fine needle aspiration biopsy of hydatid cysts. Acta Radiol. 1995; 36:168

[8]. Turkcapar A.G., Ersoz S., Gungor C., et al: Surgical treatment of hepatic hydatidosis combined with perioperative treatment with albendazole. Eur. J. Surg. 1997; 163:923

[9]. Khuroo M.S., Wani N.A., Javid G., et al: Percutaneous drainage compared with surgery for hepatic hydatid cysts. N. Engl. J. Med. 1997; 337:881

[10]. Chaudhary R, Bharti R, Shukla A. Abdominal Wall Abscess Harbouring Gall Stones and Adenocarcinoma after Laparoscopic Cholecystectomy: A Rare Case Report. Transl Biomed. 2016, 7:1.

[11]. Albayrak D, İbiş AC. Primary Hydatid Disease of Retroperitoneum: Case Report. J Clin Anal Med 2013;4(suppl 1): 87-9.

[12]. Beecherl EE. Cystic Disease of the Liver. In Zuidema GA and Yeo CJ (Eds.): Shackelford's Surgery of the Alimentary Tract, 5th ed., W. B. Saunders Company ,Philadelphia, USA. 2002,Chapter 30. 